\title{
THEMA
}

\section{Gesundheitspolitik in Großbritannien}

\section{Die Reformen der Labour-Regierung}

Schon bald nach ihrem Wahlsieg 1997 rückte Labour die Gesundheitspolitik ins Zentrum ihres Regierungshandelns. Vorausgegangen waren 18 Jahre Tory-Politik, in denen der staatliche Gesundheitsdienst NHS (National Health Service) erheblich vernachlässigt worden war. Wer sich in ärztliche Obhut begab, einen Termin, eine Diagnose oder Operation benötigte, musste vor allem mit einem rechnen: Wartezeiten. Insbesondere in seiner zweiten Amtsperiode machte Tony Blair die Reform des NHS zur Chefsache und startete ein bislang beispielloses Investitionsprogramm. Seitdem hat sich das Budget fast verdoppelt und die Organisation wie auch das Management erheblich geändert. Blairs wichtigstes Ziel dabei war, mehr Wettbewerb in die staatlichen Mammutbehörde zu bringen, in der heute allein in England 1,3 Mio. Menschen beschäftigt sind. Ob und mit welchen Mitteln dies gelungen ist, beleuchtet folgender Artikel zur Gesundheitspolitik der Labour-Regierung.

\section{Susanne Gasde}

\section{Der National Health Service als staatlich gelenkte Gesundheitsversorgung}

\section{Symbol der Solidarität}

Seit 1948 ist die britische Gesundheitsversorgung ganz in staatlicher Hand, wird überwiegend aus Steuern finanziert und bietet allen Menschen, die im Vereinigten Königreich leben, unabhängig von Nationalität und Herkunft kostenlose Behandlung. Lediglich bei Optikern, Zahnärzten und Rezepten müssen Patienten zuzahlen. Trotz aller Krisen und Wandlungen haben die Feiern zum 60. Geburtstag des NHS im Juni 2008 erneut gezeigt: Auf keine andere soziale Errungenschaft ist man im Vereinigten Königreich mehr stolz. Der NHS stiftet Identität, hält den solidarischen Geist der Nachkriegszeit lebendig, verkörpert das soziale Gewissen des Landes in Europa, in dem der Kapitalismus nie revolutionär in Frage gestellt wurde. Mögen auch manche wie Teile der Ärzteschaft das steuerfinanzierte System mit seinem heiligen Prinzip „free at the point of need“ als dauerhaft nicht finanzierbar einstufen, keine Partei würde es momentan wagen, dies in Frage zu stellen.

Susanne Gasde, Sozialreferentin an der Botschaft London
Spannung zwischen zentraler und lokaler Verantwortung

Das Department of Health managt und finanziert den NHS und muss sich dafür gegenüber dem Parlament verantworten. Die Organisation ist daher hierarchisch und durch Zielvorgaben der Politik geprägt. Seit 1999 ist das Gesundheitsministerium allein für den NHS in England zuständig, da die „Devolution“ der nationalen Politik auf die vier Länder des Vereinigten Königreiches auch die Gesundheitspolitik umfasste. Schottland, Wales und Nordirland steuern ihren NHS selbst.

Auf Initiative Tony Blairs wurde die Verwaltung mehrfach neu organisiert. Ziel war, die Versorgung mehr zu dezentralisieren und näher an den lokalen Bedürfnissen auszurichten. Ab 2002 wurden deshalb 28 regionale Gesundheitsbehörden (Strategic Health Authorities) gegründet, die im Auftrag des Ministeriums die Versorgung ihrer Region managen sollten. 2006 wurde die Zahl auf 10 reduziert, um wiederum die Effizienz zu steigern. Die Strategic Health Authorities kontrollieren heute 152 lokale NHS-Behörden, die Primary Care Trusts (PCTs). Diese bewirtschaften rund $80 \%$ des Budgets, ermitteln den Bedarf an ambulanten und stationären Leistungen vor Ort und kaufen sie entsprechend ein. Dazu schließen sie mit den einzelnen Dienstleistern wie Hausärzten, Zähnärzten oder Krankenhäusern Verträge ab. Durchschnittlich sind sie für die Versorgung von rund 300.000 Menschen verantwortlich. 
Die Gratwanderung zwischen zentraler politischer Steuerung und lokaler Verantwortung ist trotz aller Reformen schwierig geblieben. Das politisch Wünschbare ist aus Sicht des Fachpersonals vor Ort längst nicht immer deckungsgleich mit dem medizinisch Notwendigen. Zum anderen stehen britische Gesundheitsminister immer wieder unter großen Druck, die Kosteneffizienz des NHS nachzuweisen und versuchen daher, durch ministeriale Zielvorgaben diese zu steigern. Das wiederum schwächt das individuelle Verantwortungsgefühl des Personals, sei es in den Krankenhäusern oder in den PCTs. So wird häufig eher auf die Erfüllung zentraler Zielvorgaben geschaut als auf die Qualität des Services. Look out, not up - Schaut nach vorne und nicht nach oben - lautet etwa ein Ausspruch des NHS-Präsidenten David Nicholson. Mitarbeiter des Department of Health sprechen von einer tief verankerten „dependency culture“, die es zu überwinden gelte. Diese Kultur wird häufig mit dafür verantwortlich gemacht, wenn mal wieder in einem Krankenhaus skandalöse Zustände aufgedeckt und breit in der Presse berichtet werden.

\section{Die Struktur des NHS}

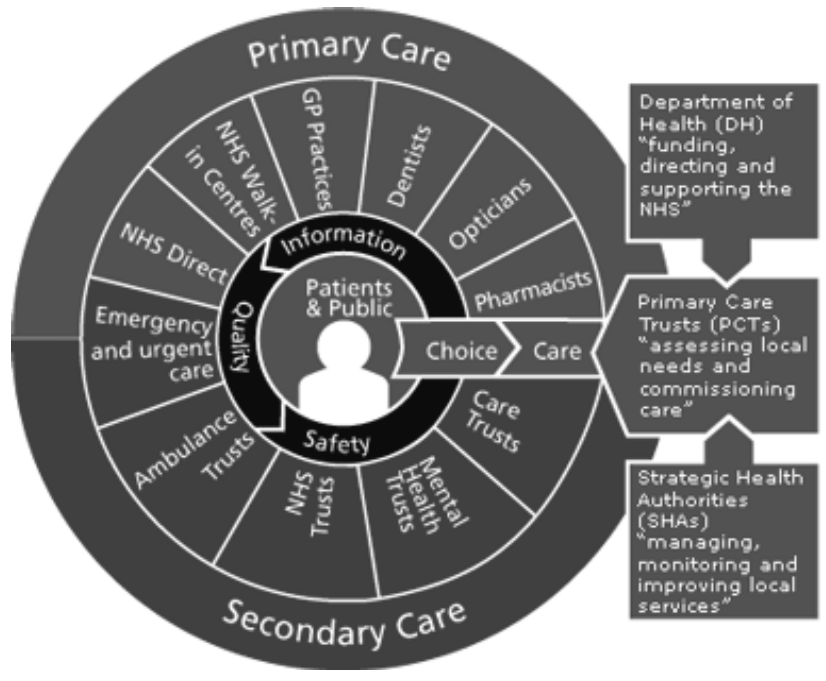

Quelle: NHS

\section{Gesundheitspolitik unter Labour}

Als Tony Blair 1997 die Regierung übernahm, befand sich der NHS in einer Dauerkrise. Im Vergleich zum europäischen Durchschnitt war die britische Gesundheitsversorgung aufgrund jahrelanger konservativer Sparpolitik mit einem BIP-Anteil von 6-7\% unterfinanziert. Gebäude samt Ausstattung waren veraltet, Personal und Medikamente knapp, Behandlungserfolge in Schlüsselbereichen wie Krebs oder Herzerkrankungen dürftig. Aus Patientensicht besonders ärgerlich waren die langen Wartezeiten: "When you ask patients what they think is wrong with the NHS, what tops the list every time is the amount of time spent waiting. Waiting for a doctor's appointment, in casualty, for test results, for an appointment with a consultant or for an operation. Most people are happy with their treatment when they receive it, but get frustrated with the length of time they have to wait." (Tony Blair 1999) Mit Start seiner zweiten Amtszeit beschloss Blair, dies mit einem gewaltigen Reformprogramm zu überwinden.

\section{Verdoppelung des Gesundheitsbudgets: "Building capacities“}

Grundlage des neuen Kurses war der sog. WanlessReport. Sir Derek Wanless, Ex-Banker und Berater Blairs, empfahl 2002 in seinem umfangreichen Bericht, den NHS durch gigantische Investitionen zu modernisieren und ihn personell und technisch besser auszustatten. Die Regierung folgte dem im Wesentlichen. Betrug 2002 der Gesundheitshaushalt noch rund $68 \mathrm{Mrd}$. GBP, sind für das Haushaltsjahr 2009/10 nun 119 Mrd. GBP vorgesehen. Diese werden überwiegend aus Steuereinnahmen finanziert. Die Einnahmen aus Beiträgen zur Sozialversicherung (National Insurance), die Arbeitgeber und Arbeitnehmer für alle Sozialleistungen zahlen - neben Gesundheit also z.B. auch für Arbeitslosengeld und Renten - betragen lediglich 98 Mrd. GBP.

In Folge des Wanless-Reports wurden jährliche Haushaltssteigerungen von mehr als 7\% üblich - ein Wachstum, das erst jüngst durch die die Finanz- und Wirtschaftskrise gestoppt wurde.

Einen großen Teil des Geldes investierte die Regierung in Personal: 7.500 neue Krankenhausärzte, 2.000 Hausärzte, 20.000 Krankenschwestern, 6.500 Therapeuten. Da zu wenig ausgebildete Kräfte vorhanden waren, rekrutierte man im Ausland. Etwa die Hälfte der eingestellten Fachkräfte hatten ihre Ausbildung nicht im Vereinigten Königreich absolviert. Bei den Ärzten waren es 60\%, bei den Krankenschwestern 90\%, die überwiegend aus dem außereuropäischen Ausland wie etwa den Philippinen, Indien, Südafrika, Zimbabwe etc. kamen. Heute sind rund 30\% der praktizierenden Ärzte Ausländer, erheblich mehr als in den 90er Jahren. Anreiz für die Zugewanderten war unter anderem eine deutliche Anhebung der Löhne nicht nur im stationären, sondern auch im ambulanten Bereich. Insbesondere Hausärzte konnten sich ab 2004 über attraktivere Vertragskonditionen mit dem NHS freuen. Sie bekamen nicht nur mehr Geld, sondern durften ihre Praxen nun auch außerhalb der üblichen Sprechstunden wie etwa abends oder an Wochenenden schließen. Für viele bedeutete das mehr Honorar für weniger Arbeit. Experten sehen darin eine Ursache dafür, dass seitdem die Notaufnahmen in den Krankenhäusern gerade an Wochenenden um ein Drittel gestiegen sind. Solche Entwicklungen nähren bis heute Zweifel, ob die enormen Investitionen der vergangenen Jahre zu einem entsprechend besseren Service geführt haben. Allein 43\% des Budgetzuwachses entfielen auf wachsende Lohn- und Rentenzahlungen. 


\section{THEMA}

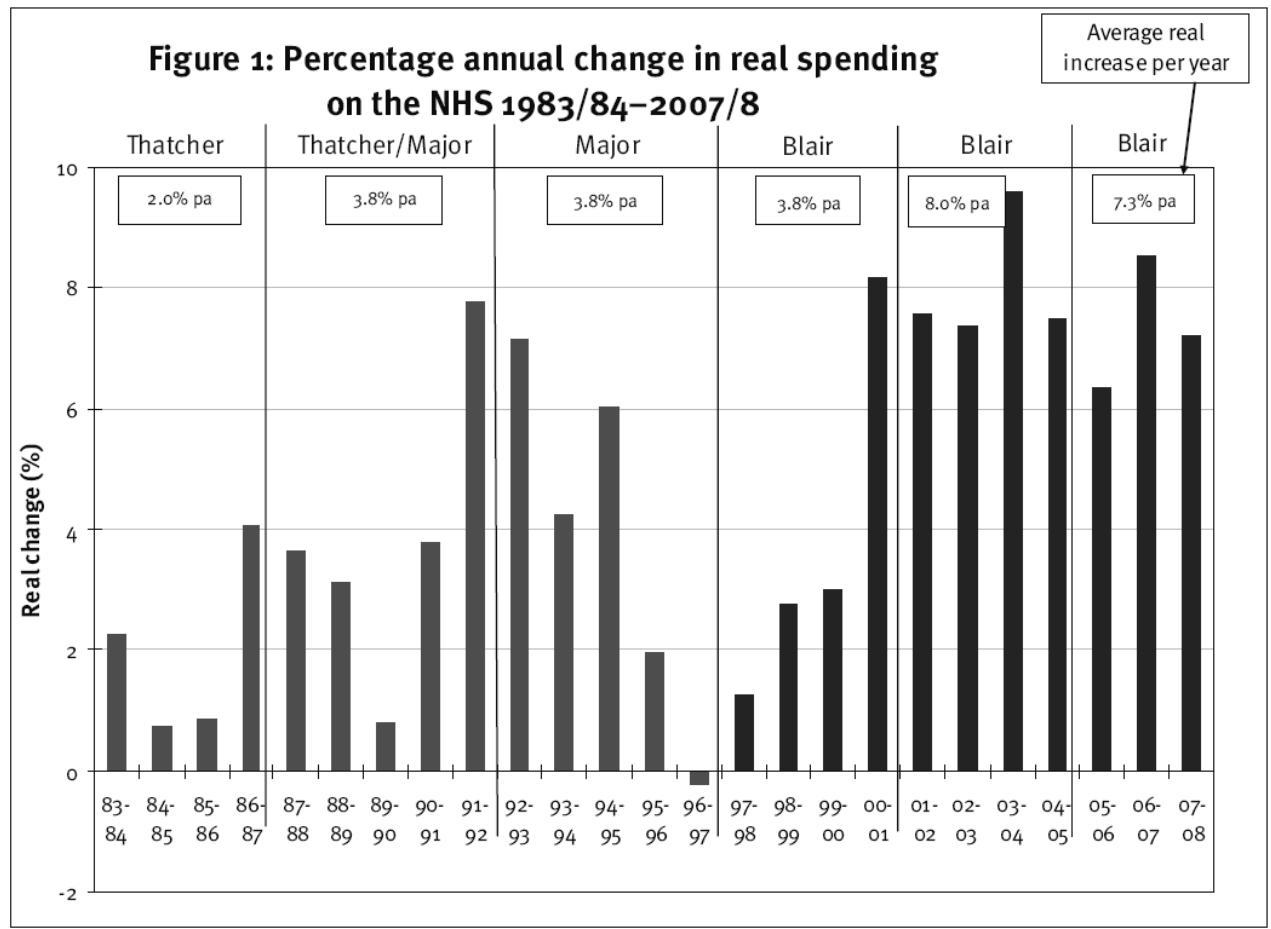

Quelle: King's Fund

Neben Personal wurde darüber hinaus in den Bau kommunaler health centres sowie Krankenhäuser investiert. Die meisten der bis 2010 geplanten 100 Neubauten wurden dabei durch öffentlich-private Partnerschaften (Private Finance Initiative) finanziert, eine Praxis, die bereits die Konservativen in den 90er Jahren eingeführt hatten. Private Unternehmen finanzieren, bauen und betreiben die Gebäude und verpachten sie anschließend an die öffentliche Hand. Allerdings gerieten die Projekte Ende 2005 ins Stocken, da teilweise am Bedarf vorbei gebaut wurde.

\section{Die wichtigsten Reformprojekte}

Tony Blair hatte den festen Willen, den NHS als ein unmittelbar nach dem II. Weltkrieg geschaffenes System an die Erfordernisse des 21. Jahrhunderts anzupassen. „The principles of the NHS are sound but its practices need to change“, hieß es. Eingeführt werden sollten:

- nationale Standards rund um die Behandlung und besserer Zugang zu Medikamenten

- Wettbewerb im staatlichen System einschließlich der Zulassung privater Dienstleister

- Leistungsanreize für mehr Qualität und Quantität u.a. durch ein neues Abrechnungssystem

- dezentraler und auf den Patienten zugeschnittener Service (Foundation Trusts)
- Wahlfreiheit für Patienten bei Arzt und Krankenhaus und besseres Informationsangebot

Neue Institutionen für Standards und Medikamentenzulassung

Neben der bereits erwähnten Verwaltungsreform wurden neue Institutionen geschaffen, um die Agenda umzusetzen und die enormen regionalen Differenzen auszugleichen. Das 1999 eingerichtete National Institute for Clinical Excellence (NICE) hat vor allem die Aufgabe, Medikamente und Behandlungsmethoden nach einer Kosten-Nutzen-Analyse zu bewerten und für die Vergabe durch den NHS frei zu geben (technology appraisals guidance). Seit 2005 ist der NHS in England und Wales gesetzlich verpflichtet, diese den Patienten zugänglich zu machen. Ebenso gibt NICE Empfehlungen zu Behandlungsmethoden und Prävention (clinical guidelines). Ziel war, die sog. postcode lottery (Postleitzahlen-Lotterie) in der Versorgung zu entschärfen, damit nicht mehr der Wohnort bzw. die Vergabepraxis des örtlichen Primary Care Trusts darüber entscheidet, ob jemand Zugang zu bestimmten Medikamenten und Behandlungsmethoden hat oder nicht.

Die Entscheidungen von NICE werden in der Öffentlichkeit von Zeit zu Zeit kritisch hinterfragt. Zuletzt, als es um die Zulassung bestimmter lebensverlängernder Medikamente für Krebskranke ging, die NICE zunächst aus Kostengründen verweigerte. Als Patienten sie dann aus eigener Tasche finanzieren wollten, wurde dies häufig von Primary Care Trusts mit dem Hinweis verwehrt, dass niemand 
Figure 2: Überblick über die Reformagenda der Labour-Regierung

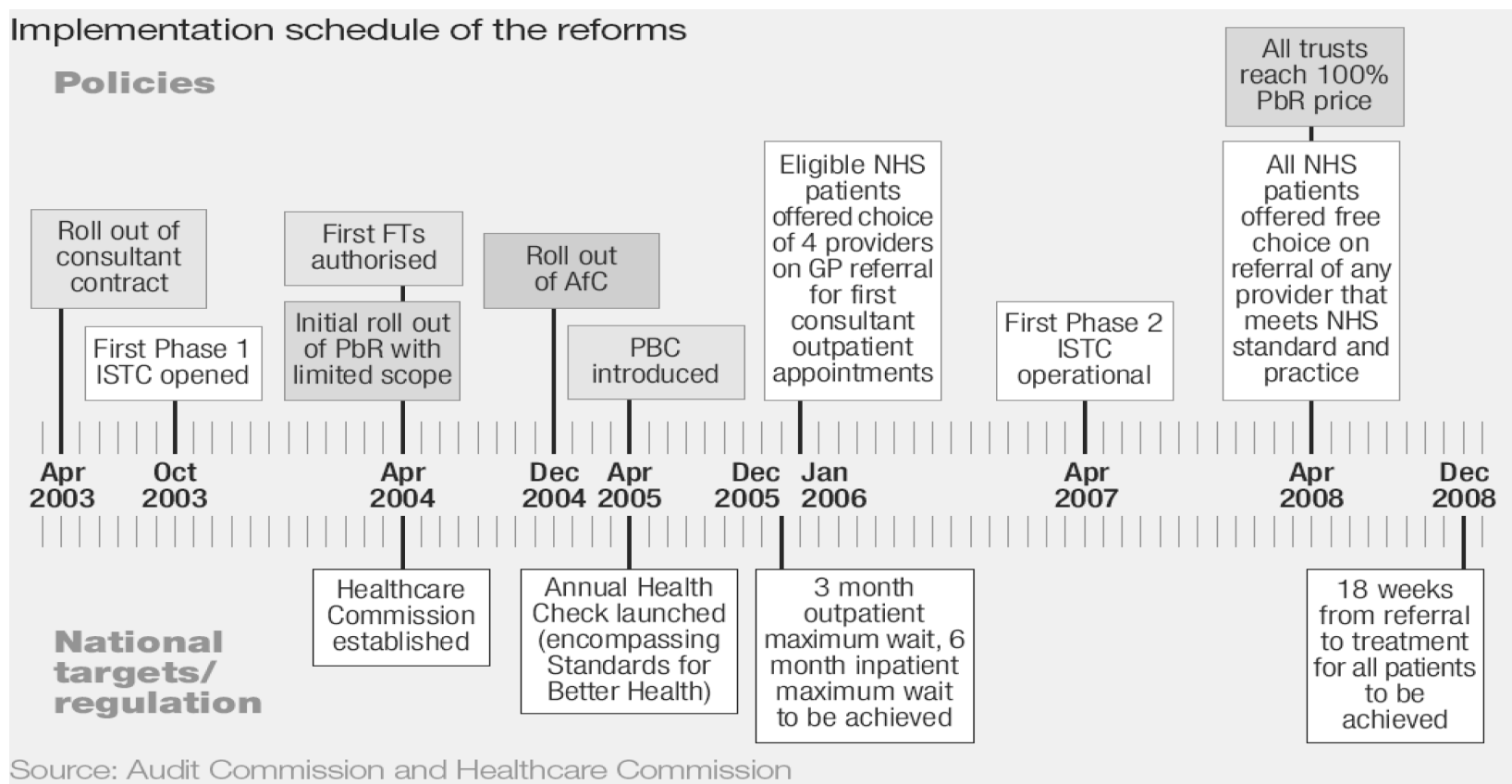

zugleich Privat- und NHS-Patient sein dürfe. Wer zuzahle, verwirke seinen Anspruch auf kostenlose Behandlung. Die Diskussion endete damit, dass unter strikten Vorgaben todkranke Menschen nun zuzahlen dürfen, ohne ihren Anspruch auf NHS- Behandlung zu verwirken. Gleichzeitig wurde die Kostengrenze für lebensverlängernde Medikamente hoch gesetzt. Ebenso wurde NICE angehalten, den teilweise bis zu zwei Jahre dauernden Entscheidungsprozess über die Freigabe neuer Medikamente auf maximal sechs Monate zu verkürzen. Insgesamt aber wird die Arbeit des Institutes sehr geschätzt. Künftig wird der Aufgabenbereich weiter ausgedehnt, da NICE ab 2010 landesweit dafür verantwortlich sein wird, einheitliche Qualitätsstandards für Behandlungsprozesse zu entwickeln und zu verbreiten.

Komplementär zu NICE wurden gleichzeitig auch nationale Standards für die Behandlung schwerer Krankheiten wie etwa Krebs, Herz- oder psychosomatische Erkrankungen sowie für die Intensivpflege bei Kindern eingeführt (National Service Frameworks).

Mit der Healthcare Commission schuf die Blair-Regierung 2004 eine weitere wichtige Einrichtung. Als unabhängige Regulierungsbehörde sollte sie auf der Grundlage von Inspektionen den Standard des geleisteten Services bewerten und die Ergebnisse veröffentlichen, Patientenbeschwerden nachgehen sowie Missstände auf ihre Ursachen hin untersuchen. Seit 2005 führt die Healthcare Commission jährliche „health checks“ durch. Dabei werden NHSEinrichtungen anhand der Kategorien „excellent“, „good“, „fair" und „weak“ geprüft, inwieweit sie die Zielvorgaben (core standards) des Gesundheitsministeriums umsetzen, etwa in Bezug auf Hygiene, Wartezeiten, Patientensicher- heit, Management. Zweifellos hat die Healthcare Commission das Bewusstsein innerhalb und außerhalb des NHS hinsichtlich Versorgungsqualität geschärft und Anreize zu Leistungsverbesserungen gesetzt. Im April dieses Jahres fusionierte sie mit der Social Care Inspection, die eine ähnliche Funktion für Einrichtungen der Pflege einnahm, zur neuen Care Quality Commission.

\section{Mehr Wettbewerb... ...durch private Anbieter}

Ein Kernanliegen von Labour war, das Monopol der NHS-Dienstleister zu brechen und erstmals durch die Einbeziehung Privater einen Anbieterwettbewerb zu schaffen. Dies betraf zunächst den stationären und diagnostischen Bereich, Notfallbehandlung ausgenommen. Die Kapazität sollte erhöht werden, um die Wartezeiten insbesondere bei geplanten Operationen auf maximal 18 Wochen zu reduzieren. Des Weiteren sollte die Wahlfreiheit der Patienten gestärkt und Innovation in der Versorgung vorangetrieben werden. Die ersten Verträge der Primary Care Trusts mit privaten Krankenhausbetreibern (Independent Sector Treatment Centres, ISTCs) wurden im September 2003 geschlossen, eine zweite Welle folgte 2005. 15\% der geplanten Operationen - so lautete das Ziel - sollten in privat betriebenen Krankenhäusern vorgenommen werden. Die marktorientierte Gesundheitspolitik Blairs stieß dabei auf durchaus hartnäckige Vorbehalte. Nicht wenige fürchteten, privates Gewinnstreben könnte auf Dauer die heiligen Prinzipien des NHS bedrohen, d.h. kostenlose Behandlung allein nach medizinischer Notwendigkeit zu gewähren und 


\section{THEMA}

nicht nach Größe des Geldbeutels. Der Widerstand innerhalb des NHS, bei den Gewerkschaften und z.T. auch innerhalb der eigenen Partei war dementsprechend deutlich. Bis heute ist die Diskussion um eine „schleichende Privatisierung“ des NHS stark präsent.

Auch wenn Untersuchungen ergaben, dass die Qualität und die Zufriedenheit von Patienten in den Privatkliniken durchaus höher ist als in NHS-Hospitälern, wurden erheblich weniger in Betrieb genommen als ursprünglich geplant. So übernahmen Privatkliniken 2007/8 mit rund 106.000 Operationen lediglich 1,8\% des gesamten Geschäftes. 5,9 Mio. Eingriffe wurden weiterhin in NHSKrankenhäusern durchgeführt. Heute sind aus der ersten Tranche noch 24 Verträge gültig, aus der zweiten lediglich 7 von 27 geplanten. Der Elan der Regierung wurde aus mehreren Gründen gebremst. Der wichtigste war sicherlich das Kostenargument. Zusätzlich zu den Investitionen in der Implementierungsphase verschlangen die ISTCs rund 11\% mehr Mittel als NHS-Krankenhäuser. Einerseits, weil man die privaten Betreiber mit garantierten Mindestpauschalen lockte, die unabhängig von der Leistung garantiert wurden, damit sie überhaupt in den Markt einstiegen. Zum anderen, weil man neu in Gebäude und Ausstattung investieren musste. Darüber hinaus bemängelten viele Primary Care Trusts, dass die gezahlten Tarife nicht die Fallzusammensetzung widerspiegelten und höher lagen als die üblichen NHS-Tarife. 2007 kam die Healthcare Commission zu dem Schluss, dass Privatkliniken tatsächlich eher die unkomplizierten Fälle von den Hausärzten überwiesen bekamen, zumal sie keine Intensivstationen vorhielten, während die schwer und kompliziert Erkrankten bei den NHS-Hospitälern landeten. Insgesamt gestaltet sich bislang die Zusammenarbeit zwischen Privatkliniken und den übrigen NHS-Einrichtungen schwieriger als bei öffentlichen Kliniken.

Die Healthcare Commission hat allerdings 2008 auch festgestellt, dass trotz des insgesamt geringen Anteils durchaus positive Effekte durch die Einbeziehung Privater zu verzeichnen seien. So habe allein die Diskussion und auch die Angst vor neuen Wettbewerbern innovative und leistungsfördernde Anreize gesetzt. Effektive und an Produktivität orientierte Methoden des Managements konnten sich so schneller innerhalb des NHS verbreiten.

Während die Regierung Brown den Ausbau der Privatkliniken erst einmal stoppte, forcierte sie im ambulanten Bereich die Zulassung privater Anbieter. Grund war vor allem die Kritik an Hausärzten (General Practitioner, GP), da diese trotz besserer Bezahlung kaum Sprechstunden außerhalb der regulären Arbeitszeiten anbieten. Primary Care Trusts haben heute die Freiheit, mit Drogerie- oder Supermarktketten über die Einrichtung neuer Arztpraxen verhandeln, die dann vertraglich auch zu Öffnungszeiten nach Feierabend verpflichtet werden.

\section{...durch Payment by Results}

Payment by Results (PbR) wurde innerhalb des englischen NHS erstmals 2003/4 eingeführt, um die Finanzströme neu zu gestalten und das System fairer und transparenter zu machen. Krankenhäuser sollten einen ökonomischen Anreiz erhalten, mehr Patienten zu behandeln und damit effizienter und ökonomischer zu handeln. Ursprüngliches Ziel war, die Zahl der stationären Behandlungen und Operationen dort zu steigern, wo es lange Wartezeiten gab. Zugleich eröffnete sich die Perspektive für das Prinzip „money follows patient choice“. Denn wenn - so die Vorstellung der Regierung - Patienten sich ihr Krankenhaus frei wählen können und über Qualität der Einrichtung gut informiert sind, können sie quasi „mit den Füßen“ abstimmen. In einem tätigkeitsbezogenen Tarifsystem sind sie die einzige Einnahmequelle. Verlieren weniger leistungsstarke Krankenhäuser ihre Einnahmen, müssen sie sich umstellen, um das Vertrauen des Patienten zurück zu gewinnen. Zuvor galten lokal ausgehandelte Blockverträge und Preise mit geringem Bezug zur tatsächlich geleisteten (Qualität der) Arbeit mit der Folge, dass die Kosten von Operationen regional stark variierten.

Mit PbR erhalten Krankenhäuser nur dann Geld, wenn tatsächlich behandelt wurde. Das Gesundheitsministerium hat seit 1998 systematisch von allen NHS-Krankenhäusern Daten über Behandlungen und ihre Kosten gesammelt und eine Liste von mehr als 1400 möglichen Eingriffen und Therapien erstellt, die jeweils mit einem Code versehen werden (Healthcare Resource Groups / HRG code). Der Preis für jeden HRG-Code bzw. für eine Behandlung wird in einem nationalen Tarif festgelegt (national tariff). Dieser spiegelt im wesentlichen die durchschnittlichen Kosten des jeweiligen Eingriffs innerhalb des englischen NHS wider. Fiktives Beispiel: Wenn eine Hüftoperation nach dem nationalen Tarif 5000 GBP kostet und eine Herzbehandlung nach einem Infarkt 2000 GBP, dann gilt das landesweit, unabhängig davon, wo operiert wurde. Wenn die Behandlung mehr kostet als der Tarif hergibt, muss das Krankenhaus selbst einen Weg finden, die Kosten zu senken. Kostet die Behandlung weniger, kann es das Geld einbehalten.

PbR wurde schrittweise eingeführt. 2005/6 waren lediglich die geplanten Operationen einbezogen. 2006/7 wurde das System auf ungeplante und Notfallbehandlungen (A\&E accident and emergency) sowie ambulante Patienten übertragen. In diesem Jahr soll die Umstellung abgeschlossen sein. Laut der Audit Commission Health, eine Art Bundesrechnungshof für das Gesundheitswesen, sind positive Effekte sichtbar: Das Honorarsystem ist fairer und transparenter geworden, Krankenhäuser arbeiten gerade bei geplanten Operationen schneller, effizienter und kostenbewusster. PbR hat zusammen mit der Gründung unabhängig wirtschaftender Kliniken (Foundation Trusts) unternehmerisches Handeln und Denken in den Mittelpunkt gerückt. Leistungsanbieter (provider) als auch Auftraggeber (commissioner) sind angespornt, ihr Management und ihre IT zu optimieren. 
Aber es gibt laut Audit Commission auch noch erhebliche Mängel: Die Qualität der Daten für die Abrechnung der Behandlungen ist oft unzureichend, sie werden nicht pünktlich und nur ungenau übermittelt bzw. erhoben. Das erschwert die Kalkulation des nationalen Tarifs. Das gegenwärtige IT-System im NHS, das für die Bearbeitung von Verträgen und Honoraren eingesetzt wird, entspricht nicht allen Anforderungen. Zudem sollte der nationale Tarif flexibler und besser auf individuelle Konstellationen herunter zu brechen sein (unbundling). Darauf verweisen auch Forschungsinstitute wie etwa der King's Fund. PbR schaffe zwar Anreize zu mehr Produktivität, helfe aber nicht, Patienten weg von der teuren Krankenhausbehandlung hinein in die wohnortnahe Gemeindebetreuung zu bekommen. Das betrifft vor allem die Nachbehandlung und Rehabilitation. Der nationale Tarif und der HRG -Code beschreiben und berechnen einen kompletten Behandlungsablauf inklusive aller administrativen und nichtklinischen Aufgaben wie Reinigung oder Essen von der ersten Terminvereinbarung an bis zum Abschluss der Nachbetreuung. Die Nachbehandlung muss jedoch nicht im Krankenhaus stattfinden, sondern kann oft auch vom preisgünstigeren kommunalen Gesundheitsservice geleistet werden. Dafür aber muss der nationale Tarif gesplittet bzw. entflochten werden (unbundling of tariffs). Da unklar ist, wer was bekommt, müssen die Primary Care Trusts zurzeit mit Kliniken und kommunalen Anbietern darüber schwierige Verhandlungen führen. Kliniken jedenfalls haben per System keinerlei Anreiz, im Sinne einer integrativen Pflege mit den kommunalen Anbietern zu kooperieren. Die Möglichkeiten, nationale Tarife zu entflechten, ist also eine Aufgabe, die es noch zu lösen gilt.

\section{...durch neue Formen des Managements}

Seit 2004 können besonders leistungsstarke stationäre Einrichtungen den Status eines "Foundation Trust" bzw. "Foundation Hospital" erlangen. Dies ist für Krankenhäuser attraktiv, weil sie dann unabhängig vom Gesundheitsministerium und den Strategic Health Authorities wirtschaften und planen dürfen. Sie bleiben Teil des NHS, bekommen ihr Budget aber nicht von der Zentrale zugeteilt. Je nach Kapazität können sie sich auch selbst Kapital beschaffen und Überschüsse neu investieren. Foundation Trusts sind damit auch ein Instrument, die lokale Verantwortung zu stärken. Rechtlich gesehen agieren sie als unabhängige juristische Personen (Public Benefit Corporations) und müssen sich u.a. gegenüber einem Bord of Govenors verantworten, in dem Vertreter von Patienten, Personal und Kommune sitzen. Bewerben dürfen sich alle Krankenhäuser mit Operationsbetrieb, Spezialkliniken sowie psychosomatische Einrichtungen, die die nationalen Standards der Regierung erfüllen. Demnächst sollen auch Ambulanzdienste einbezogen werden. Mittlerweile haben sich von 239 Einrichtungen 121 den Status eines Foundation Trust erworben.

\section{...und der Auftragsvergabe}

Die Marktorientierung der Verwaltungsbeziehungen im britischen Gesundheitswesen kommt in dem Begriff „commissioning“ (Auftragsvergabe) zum Ausdruck, der die staatlichen Einkäufer von den Anbietern der Gesundheitsdienstleistungen trennt. Primary Care Trusts haben die Aufgabe, den Bedarf ihrer Region an Dienstleistungen zu ermitteln und diese dann gemäß der selbst gesetzten Prioritäten und nationalen Zielvorgaben einzukaufen. Über allem steht dabei die Erwartung, wo immer möglich Patienten wohnortnah und außerhalb von Krankenhäusern zu behandeln. In diesem Punkt allerdings ist das Management der Primary Care Trusts insgesamt wenig erfolgreich. Die weit überwiegende Mehrheit ambulanter Behandlungen findet immer noch in Krankenhäusern statt. Als ursächlich gelten nicht nur die für Patienten ungünstigen Öffnungszeiten der Hausarztpraxen, sondern auch fehlende Vorsorgeuntersuchungen sowie die unzureichende Betreuung chronisch Kranker. Rund 15 Millionen Menschen gehören in England dazu. Sie machen etwa 55\% der Hausarztbesuche aus, 68\% der Besucher in den Notaufnahmestellen der Krankenhäuser (A\&E attendances) sowie 77\% der Krankenhausbelegungen. Würden sie besser und konstant von einem Arzt begleitet, könnte in vielen Fällen die Einweisung in ein Krankenhaus vermieden werden. Dies sicher zu stellen ist auch Aufgabe der Primary Care Trusts.

Die Regierung hat vielfältige Initiativen gestartet, um die Bedarfsermittlung und Auftragsvergabe durch die PCTs zu professionalisieren und ihre analytischen und planerischen Fähigkeiten zu steigern. Mit mäßigem Erfolg. Dies führen Experten auch auf die vielen Verwaltungsreformen zurück, die den Strategic Health Authorities und den Primary Care Trusts immer wieder neue Einzugsbereiche bescherten, zuletzt 2006.

$\mathrm{Zu}$ den jüngeren Initiativen gehört das sog. Practice Based Commissioning. So wird seit 2005 versucht, insbesondere Hausärzte mit in den Ausschreibungs- und Vergabeprozess einzubinden. Ein entsprechendes zusätzliches Budget soll sie anspornen, Überweisungen effektiver zu managen und mit zu helfen, örtliche Angebote kostengünstiger zu gestalten. Doch die Mehrheit der Ärzte lässt sich bislang noch nicht auf diese Doppelrolle als Anbieter (provider) und Einkäufer (commissioner) ein. Ein weiteres Manko ist zudem der Mangel an verlässlichen Daten über Behandlungsergebnisse, was eine qualitätsorientierte Auftragsvergabe erschwert. Immerhin: Die Einführung der tätigkeitsbezogenen Honorierung mit Payment by Results hat die Verhandlungsposition der Primary Care Trusts insgesamt gestärkt.

\section{...durch mehr Wahlfreiheit für Patienten}

Eines der wichtigsten Instrumente, um die Qualität der Gesundheitsversorgung zu steigern, ist aus Sicht der Regierung die Wahlfreiheit des Patienten (patient choice). Dieser wird - so die Erwartung - keine Einrichtungen mit schlechtem Service aufsuchen und so deren Wettbewerb 


\section{THEMA}

untereinander forcieren. Seit 2008 können Patienten ihr Krankenhaus nun vollkommen frei wählen - eine Entscheidung, die noch zwei Jahre zuvor ausschließlich von Ärzten getroffen wurde. Nach und nach werden weitere Bereiche in die choice policy einbezogen wie etwa die freie Wahl des Hausarztes oder die Behandlung chronisch Kranker. So sollen nächstes Jahr Modellprojekte gestartet werden, um persönliche Budgets für die rund 15 Mio. chronisch Kranken in England zu erproben. Vorbild sind dabei entsprechende Pilote in der Pflege (social care), die erfolgreich verlaufen sind. Dabei erhalten Patienten eine bestimmtes Budget, um sich selber Dienstleistungen wie etwa Physiotherapie oder ambulante Pflege einzukaufen. Grundlage dafür ist ein zuvor mit dem Arzt vereinbarter Heilungsplan. Das Gesundheitsministerium hofft, damit die Position des Patienten zu stärken und ihm mehr Kontrolle über Behandlung und Anbieter zu geben.

Alle Experten sind sich einig, dass größere Mitbestimmung des Patienten grundsätzlich eine wichtige Triebfeder für Innovation ist. Doch ist bislang nicht nachweisbar, dass sich tatsächlich Patientenströme verändert haben. Zum einen braucht eine solche Politik Zeit, bis sie wirkt, zum anderen sind längst noch nicht alle Voraussetzungen geschaffen. Es mangelt weiterhin an der entsprechenden Infrastruktur etwa bei stationären Einrichtungen, die bereits als qualitativ gut eingestuft wurden. Private Kliniken sind rar, lediglich die Hälfte der NHS Einrichtungen haben in England den Status eines Foundation Trust erreicht. Außerdem sind die Bewertungen der Kiniken durch die Regulierungsbehörde oft so allgemein, dass einzelne Dienstleistungen kaum herauszufiltern sind. Systematisch aufbereitete Daten über Erfolge und Qualität von Behandlungsmethoden und Einrichtungen gibt es nicht. Zudem ergaben Untersuchungen der Healthcare Commission Ende 2007, dass lediglich 44\% der Patienten in England überhaupt Alternativen insbesondere durch ihren Hausarzt angeboten bekamen. Eine Reihe von Patienten bevorzugt grundsätzlich eine wohnortnahe Einrichtung, unabhängig davon, ob diese gerade im jährlichen „health check“ schlecht bewertet wurde. Allerdings lassen Umfragen auch auf eine allmähliche Prioritätenverschiebung bei Patienten schließen. So überholt bei manchen Befragungen das Kriterium Sauberkeit allmählich das der Wohnortnähe, was auch an der umfangreichen Berichterstattung über steigende Krankenhausinfektionen liegen mag.

\section{Die Qualitätsoffensive unter Gordon Brown}

Mehr Wettbewerb und größere Patientenbeteiligung prägen auch die Gesundheitspolitik der Regierung Brown. Ins Zentrum der Agenda wurde allerdings ein weiterer Aspekt gerückt: Qualität. Sollte Payment by Results vor allem die Produktivität steigern, geht es nun die systematische Ausrichtung aller Leistungen des NHS an universellen Qualitätskriterien und ihrer flächendeckenden Kontrolle.

Qualität umfasst dabei drei Aspekte: Safety, Effectiveness, Patient Experience. Zurzeit arbeitet das National Institute for Health and Clinical Excellence (NICE) zusammen mit dem Gesundheitsministerium Kriterien für qualitativ gute Behandlungsprozesse aus bzw. trägt bestehende zusammen. Ab Herbst werden diese in ausgewählten Einrichtungen getestet. Im April 2010 erhält dann NICE die volle Verantwortung dafür, neue Standards zu entwickeln und zu verbreiten. Ein neu gegründeter National Quality Board soll als eine Art Supervisor die Erstellung und Einhaltung der neuen Qualitätsstandards national überwachen, die Arbeit von NICE bewerten und Schwerpunkte setzen. Mitglieder sind Experten von den wichtigsten gesundheitspolitischen Institutionen wie u.a. der Chief Executive und der Medical Director des NHS, Chair of NICE, Chair of Care Quality Commission, Chair of National Patient Safety Agency sowie Verbandsvertreter der Ärzte und Krankenschwestern. Der erste Bericht wird im Herbst erscheinen.

Bislang gibt es kaum Hinweise des Gesundheitsministeriums, wie die Qualität klinischer Prozesse künftig gemessen werden soll. Zum einen soll Bestehendes gesammelt, zum anderen neue Kriterien entwickelt werden. Als Vorbild gelten die Pilote „Advancing Quality“, die der NHS North West zurzeit in 40 Kliniken erprobt. Dort konzentriert man sich auf fünf Bereiche: Herzinfarkt, Herzinsuffizienz, Bypassoperation, Hüft- und Knieersatz, Lungenentzündung. Für jeden Bereich wurden 34 Standards gesetzt, um die Behandlungsqualität zu messen. Der NHS North West prognostiziert, so pro Jahr rund 150 Leben retten zu können und 17 Mio. GBP einzusparen, da Rücküberweisungen vermieden, Krankenhausaufenthalte verkürzt und Komplikationen minimiert würden.

Sind einmal allgemeingültige Kriterien gefunden, sollen neue Instrumente entwickelt werden, vorrangig in Form neuer IT-Software (clinical dashboards), mit denen die Einrichtungen ihre Leistungen überprüfen können. Die Entwicklung dieser Software ist allerdings extrem teuer und wird einige Jahre in Anspruch nehmen.

Neu ist der Wille, Qualität auch durch Patienten bewerten zu lassen. PROMs ist das Stichwort und steht für patient-reported outcome measures. So werden seit April dieses Jahres systematisch Rückmeldungen der Patienten mittels eines einheitlichen Fragebogens erhoben, der unmittelbar vor und nach der Behandlung auszufüllen ist. Dabei wird nicht nur die subjektive Zufriedenheit abgefragt, sondern auch Aspekte der Lebensqualität, Mobilität und des psychischen Zustandes. Grundlage sind zunächst die Erfahrungen bei Operationen an Hüfte, Knie, Krampfadern und Knochenbrüchen. Schätzungsweise 250.000 Patienten werden in England befragt, die Ergebnisse sind später auf der NHS Choices Website zu veröffentlichen. Die Bedeutung dieses Verfahrens wird von einigen Experten hoch eingeschätzt. So meint etwa der King's Fund, PROMS könne die manchmal kruden, oft auch irreführenden Methoden der rein aktivitätsbezogenen Messung von Qualität korrigieren. Ebenso werde es so den Primary Care Trusts erleichtert, leistungsfähige Anbieter zu identifizieren. Andere sind vorsichtiger, insbesondere was die Kosten betrifft.

Darüber hinaus werden zurzeit alle Einrichtungen des NHS gesetzlich verpflichtet, ab April 2010 regelmäßig „Quality Accounts“ an die Primary Care Trusts abzuliefern, 


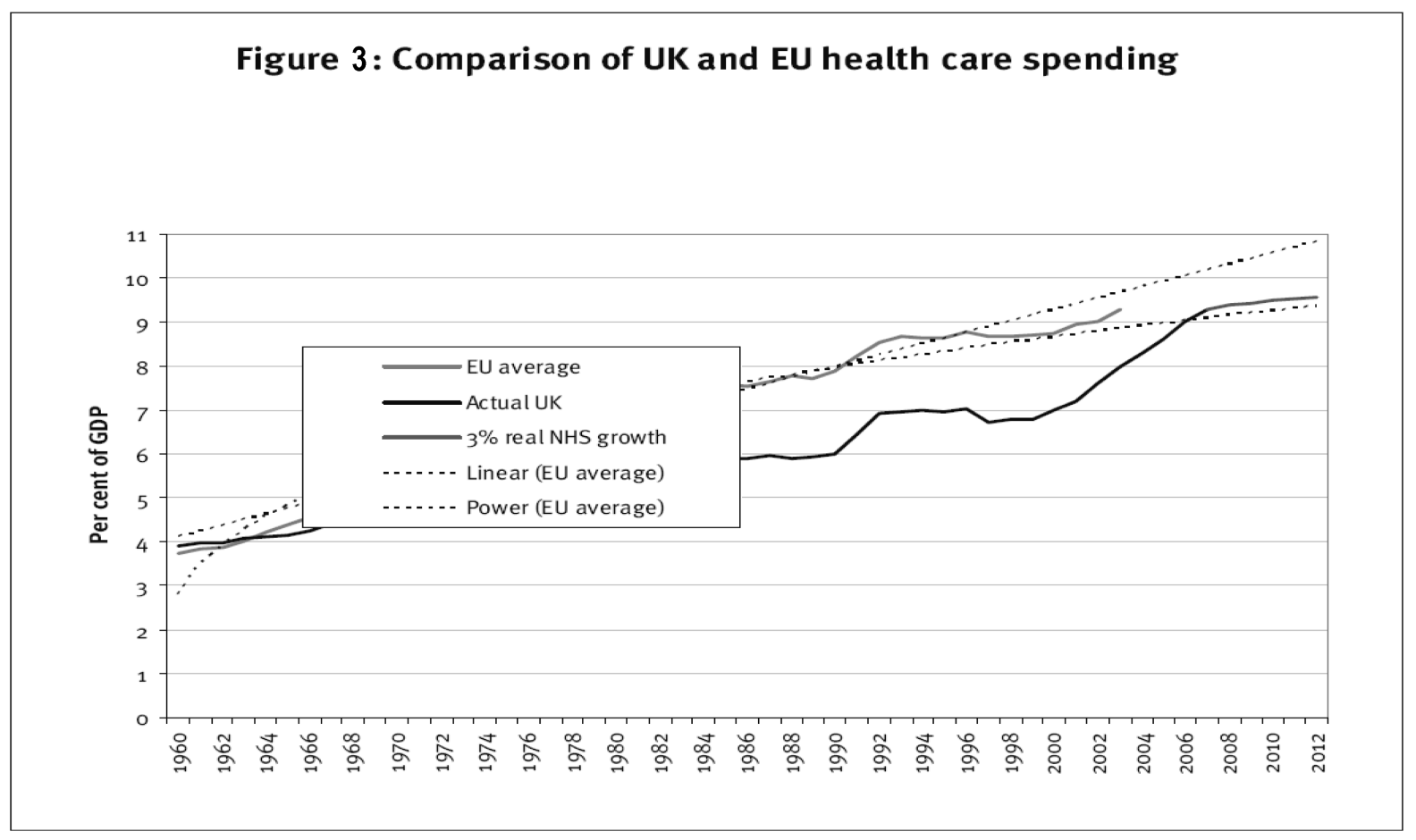

Quelle: King's Fund

ähnlich ihrem jährlichen Budgetbericht. Nach gegenwärtigem Verständnis sollen sich diese Berichte vorrangig an lokalen Prioritäten ausrichten, aber auch Rechenschaft über die Einhaltung nationaler Standards geben. Diese Informationen werden von der Care Quality Commission gesammelt und bewertet. Krankenhäuser müssen dann etwa berichten, wie viele Patienten nach Operationen gestorben sind, welche Methoden erfolgreich und ob die Patienten zufrieden waren. Rund 50 Operationsarten werden künftig ausgewertet, erheblich mehr als heute. Jede Hausarztpraxis und jede Klinik soll so aufgrund vergleichbarer Daten ihre Leistungen im Verhältnis zu anderen einordnen können. Darüber hinaus sollen Primary Care Trusts künftig Einrichtungen für gute Leistung belohnen. Das Gesundheitsministerium will allerdings zunächst den Anteil der erfolgbezogenen Honorierung gering halten und auf maximal $0,5 \%$ des Jahreseinkommens begrenzen.

Medien, Fachwelt und Patientengruppen begrüßen im Wesentlichen die Vorschläge, meistens allerdings mit dem Hinweis darauf, dass konkrete Aussage zu Kosten und Finanzierung fehlen und die Umsetzbarkeit erst abzuwarten bleibt. Das ist verständlich. Das Worst Case Scenario wäre die Entstehung einer lähmenden Bürokratie infolge einer neuen, im Detail zentral gesteuerten Qualitätskontrolle mit Institutionen, die sich gegenseitig behindern. Bislang ist nicht klar, in welchem Verhältnis NICE, die neue Care Quality Commission und der Quality National Board zueinander stehen werden.

\section{Fazit und Ausblick}

\section{Bewertung der Reformpolitik}

Anders als zu Zeiten der Konservativen hat die Gesundheitspolitik durch die Labour-Regierung deutlichen Auftrieb erfahren. Nie zuvor wurde personell und finanziell in einem solchen Ausmaß investiert, so dass die britische Gesundheitsversorgung insgesamt sich dem Niveau der europäischen Nachbarn deutlich angenähert hat.

Das war zweifellos ein mutiger Schritt, doch hat sich die Verdopplung des Gesundheitsbudgets ausgezahlt? In der britischen Öffentlichkeit wird diese Frage nachdrücklich gestellt. Das Urteil: ja und nein. So auch das von Derek Wanless, auf dessen Empfehlungen hin die gigantischen Investitionen erfolgten. Das zentrale Problem der Wartezeiten sei zwar gemildert, das Personal enorm aufgestockt und Krankheiten wie Krebs und Herzleiden erfolgreicher bekämpft sowie Praxen und Kliniken besser ausgestattet worden. Jedoch seien die vielen organisatorischen Neuerungen innerhalb des NHS überhastet, ohne ausreichende Folgeabschätzungen und mit hohem Ressourcenverlust durchgeführt worden. Zentrale Zielvorgaben wie etwa die, jeden Patienten in der Notaufnahme innerhalb von vier Stunden zu behandeln, hätten teilweise falsche Anreize gesetzt und nicht zu mehr Qualität geführt. Die Weisung an Hausärzte, Termine innerhalb von 48 Stunden zu gewähren, habe zeitweise bewirkt, dass vielerorts keine längerfristigen Vorbuchungen möglich gewesen seien.

Vor allem aber, so Wanless, habe es die Regierung nicht vermocht, die Menschen für einen gesünderen Lebensstil 


\section{THEMA}

zu gewinnen. Insbesondere die rasant steigende Fettleibigkeit müsse mehr im Fokus stehen, da sie auch finanziell die größte Herausforderung der Zukunft darstelle. Diese Forderung wird gestützt durch jüngste regierungseigene Prognosen, die 2050 rund 60\% der Männer, 50\% der Frauen und $25 \%$ der Kinder als fettleibig sehen, falls nicht mehr unternommen werde. Bereits jetzt steht das Vereinigte Königreich bei steigender Tendenz in punkto Fettleibigkeit auf Platz zwei hinter den USA.

Anteil der Erwachsenen nach Alter und Geschlecht, die übergewichtig und fettleibig sind:

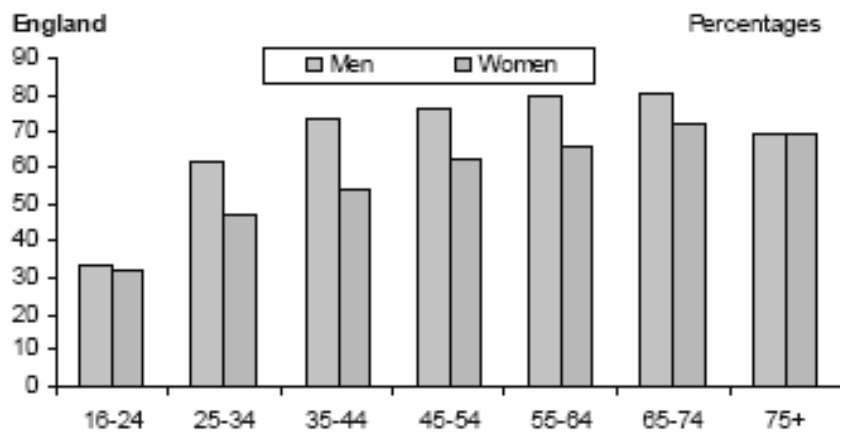

Quelle: NHS, Health Survey for England 2006

Tatsächlich fällt auf, dass Briten im europäischen Vergleich mit am wenigsten gesundheits- und körperbewusst leben. Die meisten Krankheiten entstehen, weil entweder zu viel getrunken, gegessen oder geraucht wird. Die Zahl chronisch Kranker wächst stetig.

Die Beurteilung der Reformpolitik Labours fällt deshalb bei so vielen Experten ambivalent aus, weil die enormen Ausgabensteigerungen immer wieder mit den weiterhin bestehenden Defiziten kontrastiert werden. So gibt es beispielsweise immer noch Krankenzimmer, in denen Frauen und Männer gemeinsam untergebracht sind. Insbesondere ältere Patienten beschweren sich auch heute noch über mangelhafte Pflege und Unterbringung in Krankenhäusern, über fehlende Hilfe bei der Körperhygiene, ungemachte Betten oder schlampige Reinigung. Berichte über hohe Infektionsraten in Krankenhäusern oder über allgemein katastrophale Zustände trüben immer wieder das offizielle Wunschbild eines „Gesundheitssystems von Weltklasse“. Sie werfen jedes Mal die Frage auf, inwieweit es sich tatsächlich um Einzelfälle handelt. Jüngstes Beispiel: Krankenhaus Mid Staffordshire. Im März 2009 veröffentlichte die Healthcare Commission ihren Untersuchungsbericht über die dortige Situation, die noch vor einem Jahr zu vermeidbaren Todesfällen führte. Folgende Auszüge belegen das Ausmaß der Missstände:

- In der Notfallaufnahme wurden unqualifizierte Hilfskräfte an der Rezeption beschäftigt, die die Dringlichkeit einer Behandlung beurteilen sollten. Ein Patient etwa musste mit einer offenen Fraktur am Ellbogen mehr als vier Stunden warten, blutig und ohne Schmerzmittel.
- Personalmangel bei Ärzten und Krankenschwestern führte u.a. dazu, dass es keine Bereitschaftsdienste für Fachärzte gab oder Patienten nicht betreut wurden, etwa wenn sie auf Toilette mussten oder unter akuten Schmerzen litten. Allein 17 Krankenschwestern fehlten in der Notaufnahme.

- Krankenschwestern waren unzureichend ausgebildet, konnten teilweise keine Transfusionen legen oder Kardiogramme lesen.

- Notwendige Operationen wurden regelmäßig verzögert, besonders für Traumapatienten an Wochenenden.

- Patienten bekamen keine blutverdünnenden Mittel vor Operationen, was zu Thrombosen mit anschließender Todesfolge führte.

Spricht man Ministeriumsmitarbeiter darauf an, wird auf Fehler im obersten Management hingewiesen. Der Trust habe finanzielle Probleme gehabt und sei durch die Einführung des Nationalen Tarifs im Rahmen von Payment by Results gezwungen gewesen zu sparen. Dabei sei man so versessen darauf gewesen, die Zielvorgaben des Ministeriums zu erfüllen, um unabhängiger zu werden, dass offensichtliche Mängel in Kauf genommen wurden. Tatsache ist, dass die Probleme bereits Jahre bestanden, ohne von den örtlichen Behörden bemerkt zu werden.

In dieses Bild passt, was die Healthcare Commission in ihrem letzten Bericht 2008 festgestellt hat. Lediglich die Hälfte der NHS Einrichtungen erfüllen die von der Regierung festgelegten Grundstandards (core standards), insbesondere hinsichtlich Hygiene. Welche Folgen die genannten Mängel für die Versorgungsqualität und die Sicherheit der Patienten in der Breite hat, lässt sich mangels systematisch erhobener Daten über Behandlungsergebnisse nicht abschließend beurteilen. Die Healthcare Commission hatte aus diesem Grund die Einrichtung einer entsprechenden nationalen Datenbank gefordert.

Trotzdem: Wenn man sich vor Augen führt, an welchem Punkt Labour gestartet ist, überwiegt eindeutig das Positive in der Bilanz. Ob Wartezeiten, nationale Standards, personelle und technische Ausstattung, regionale Unterschiede oder die Behandlung schwerer Krankheiten - viele können sich heute nicht mehr erinnern, wie düster es hier 1997 ausgesehen hat und wie weit das Vereinigte Königreich vom Standard benachbarter Länder entfernt war. Labour hat den gewagten Beschluss gefasst, eine so gewaltige Organisation wie den NHS innerhalb weniger Jahre von Grund auf reformieren zu wollen. So gesehen wundert es wenig, dass das Gesamtbild noch so uneinheitlich ist, zumal die Veränderungen erst langsam vom Personal akzeptiert und verinnerlicht werden.

\section{Die Zukunft}

Nachdem jahrelang das Gesundheitsministerium mit regelmäßig satten Haushaltssteigerungen verwöhnt wurde, wird künftig nun auch der NHS die Auswirkungen der 
Finanz- und Wirtschaftskrise spüren. Das stellt den neuen Gesundheitsminister Andy Burnham genauso wie seine möglichen Nachfolger nach der Wahl im nächsten Jahr vor die schwierige Aufgabe, das proklamierte Ziel von mehr Qualität mit weniger Geld umzusetzen. Bereits jetzt muss dass Department of Health bis 2010/11 auf Wunsch des Finanzministers 2,3 Mrd. GBP einsparen. Doch in den nächsten Jahren werden die Einschnitte voraussichtlich tiefer gehen. Die neue Sprachregelung lautet nun: Mehr Qualität und Patientensicherheit gehen Hand in Hand mit mehr Leistung für weniger Geld (better value for money). Doch im streng nach Zielvorgaben arbeitenden System bedeutet dies eine enorme Belastung für die Verantwortlichen vor Ort. Für die Krankenhäuser etwa wird der Druck steigen, den Aufenthalt von Patienten zu verkürzen. Die Primary Care Trusts werden noch mehr angehalten sein, stationäre Überweisungen so weit es geht zu vermeiden, was den Zugang zu Spezialisten weiter erschweren kann. Auch die Vergabe teurer Medikamente wird künftig noch restriktiver, das Ministerium etwa hofft auf Einsparungen durch Verzicht auf Markenprodukte.

Je nachdem, wie hoch die Einschnitte in Zukunft sein werden und ob eine konservative Regierung gewählt wird, sind Schritte in Richtung Gebührenzahlungen für Dienstleistungen des NHS oder zusätzliche Elemente eines privaten Versicherungssystems mit erlaubten Zuzahlungen nicht auszuschließen.

\section{Literaturauswahl:}

Appleby, John: Counting what matters most to patients. The King's Fund. Februar 2009

Audit Commission: The right results? Payment by Results 2003-2007. Februar 2008

Department of Health: The NHS Plan. A plan for investment. A plan for reform, Juli 2000

Department of Health: Health Bill 2009. Briefing Sheet

Department of Health: High Quality Care for All - NHS Next Stage Review Final Report. 2008

Healthcare Commission / Audit Commission: Is treatment working? Progress with the NHS system reform programme. Juni 2008

Healthcare Commission: State of Healthcare, Dezember 2008

House of Commons, Health Committee: NHS Next Stage Review, First report of Session 2008-9

House of Commons, Health Committee: Health Inequalities. Third Report of Session 2008-9

King's Fund: Payment by Results. Briefing. Oktober 2008

King's Fund: Patient Choice. Briefing. Januar 2008

Seldon, Anthony (Hrsg.): Blair's Britain 1997-2007. Cambridge 2007

Thorlby, Ruth / Maybin, Jo: Health and 10 years of Labour government: achievements and challenges. King's Fund Briefing. Mai 2007

Wanless, Derek: Securing our future health: taking a long-term view: final report. April 2002

Wanless, Derek: Our future secured? A review of NHS funding and performance. September 2007

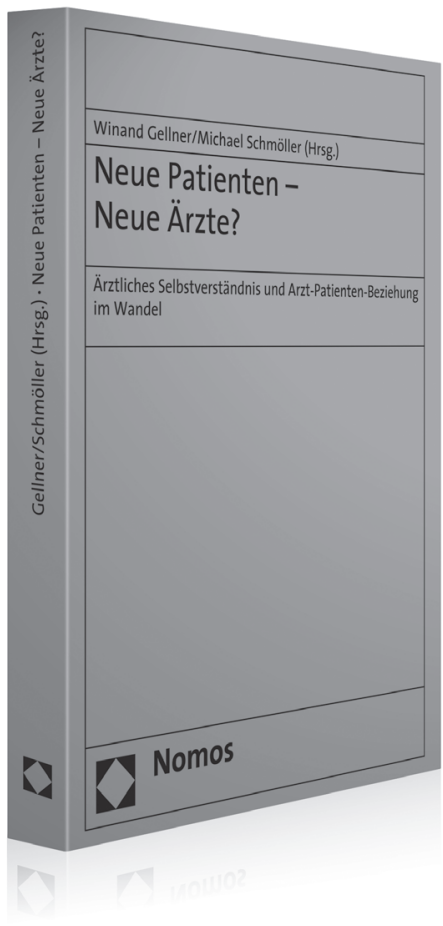

Bitte bestellen Sie im Buchhandel oder versandkostenfrei unter $\downarrow$ www.nomos-shop.de

\author{
Neue Patienten - Neue Ärzte? \\ Ärztliches Selbstverständnis und Arzt-Patienten-Beziehung im Wandel \\ Herausgegeben von Prof. Dr. Winand Gellner und Michael Schmöller \\ 2008, 224 S., brosch., 39,- $€$, \\ ISBN 978-3-8329-3570-2
}

Der vorliegende Band widmet sich dem durch die jüngsten gesundheitspolitischen Reformmaßnahmen hervorgerufenen Wandel im Arbeitsalltag und Selbstverständnis des Arztes sowie im Verhältnis zu seinen Patienten. Den Ausgangspunkt bilden die Ergebnisse einer repräsentativen Ärzteumfrage durch das »Zukunftsforum Gesundheitspolitik» (Zufog).

"sollte das Buch sich in jeder einschlägigen Bibliothek wiederfinden, sowohl als materialreiche Quelle als auch zur Anregung für weitere wissenschaftliche und gesundheitspolitische Aktivitäten."

Prof. Norbert Schmacke, G+S 2/o9 